\title{
Power Spectrum Characterization of Systematic Coded UW-OFDM Systems
}

\author{
Morteza Rajabzadeh*†, Heidi Steendam*, Hossein Khoshbin ${ }^{\dagger}$ \\ *DIGCOM Research Group, TELIN Dept., Ghent University, Ghent, Belgium \\ ${ }^{\dagger}$ Electrical Engineering Department, Ferdowsi University of Mashhad, Mashhad, Iran
}

\begin{abstract}
Unique word (UW)-OFDM is a newly proposed multicarrier technique that has shown to outperform cyclic prefix (CP)-OFDM in fading channels. Until now, the spectrum of UWOFDM is not thoroughly investigated. In this paper, we derive an analytical expression for the spectrum taking into account the DFT based implementation of the system. Simulations show that the proposed analytical results are very accurate. Compared to CP-OFDM, we show that UW-OFDM has much lower out-ofband (OOB) radiation, which makes it suitable for systems with strict spectral masks, as e.g. cognitive radios. Further, in this paper, we evaluate the effect of the redundant carrier placement on the spectrum.
\end{abstract}

\section{INTRODUCTION}

Multicarrier techniques, especially orthogonal frequency division multiplexing (OFDM), have been adopted as the main signaling schemes for most wireless communications standards due to their ability to counteract the effects of time dispersive channels, the simplicity they offer in terms of equalization, flexibility for achieving different data rates and having flexible spectral characteristics. OFDM is a blockwise transmission scheme in which a number of data symbols are modulated using an inverse discrete Fourier transform (IDFT). When a high data rate signal is passed through a timedispersive channel, intersymbol interference (ISI) occurs. In OFDM, a time guard interval is inserted between the consecutive OFDM frames to compensate for the ISI. Different guard intervals can be found in the literature such as cyclic prefix (CP), zero padding (ZP) and known symbol padding (KSP) [1], [2] out of which $\mathrm{CP}$ is more utilized in practical systems. Recently, a new family of OFDM systems named UW-OFDM is proposed, in which the time guard interval is part of the IDFT block [3]. In a two-step implementation of UW-OFDM systems, first a block of zeros is constructed at the tail of the IDFT output. This can be done by two approaches [4]: 1) by appropriately loading a set of redundant carriers in systematic coded UW-OFDM system, i.e. data carriers and redundant carriers are separated, and 2) by distributing the redundancy over all active subcarriers by using a code generator matrix in non systematic coded UW-OFDM system. Then, in the second step, the zero part of the block is filled with the known unique word samples. Like $\mathrm{CP}$, the added unique word transforms the linear convolution of the transmitted signal with the channel impulse response into a cyclic convolution. This allows for a low complexity equalization at the receiver. Contrary to the $\mathrm{CP}$ which is random, the unique word is deterministic and can be designed for some practical needs such as synchronization or channel estimation. Although the UW-OFDM frame is shorter than CP-OFDM and contains lower number of data symbols, the bit error rate and throughput of UW-OFDM is comparable to those of CP-OFDM [3], [4].

The spectral behavior is an important issue in multicarrier systems, as it is known that OFDM based systems have high sidelobes. As in practice, transmission schemes must meet some regulatory spectrum masks, it is of importance that the in-band and out-of-band radiation of the system is controlled. For CP-OFDM, much research has been devoted to this topic. However, most of the studies which require the spectral model have considered a model that is based on the analog implementation of the multicarrier systems, as e.g. for spectrum modeling [5], blind carrier tracking [6], in-band and out-of-band radiation reduction and sidelobe suppression [7], [8], [9], [10], [11]. Although this analytical model is easy to use, it is not precise for practical systems, in which multicarrier transmitters are typically implemented by an IDFT followed by a digital-to-analog converter, and the power spectral density of this implementation is generally not equivalent to that of the analog implementation. Conditions for equivalency of these approaches are discussed in [12]. The spectral characteristics of CP-OFDM based on the DFT based implementation is investigated in [1], [13], [14].

To our best knowledge, the spectrum for UW-OFDM is only considered in [4], where the authors claim that UWOFDM has lower out-of-band radiation than CP-OFDM. These results were based on simulations only. In this paper, we derive the analytical expression for the power spectral density of systematic coded UW-OFDM system using the DFT based implementation. In order to obtain an expression for the spectrum of CP-OFDM systems, in [1], it is assumed that the symbols transmitted on the carriers are independent. This assumption is not applicable to UW-OFDM, where the redundant carrier symbols are a linear combination of the data symbols, introducing correlation between the subcarriers. Fortunately, this correlation is repeated in the successive UWOFDM symbols. So, the sequence at the output of the UWOFDM is cyclostationary. We use this property to derive novel expressions for the spectral behavior of UW-OFDM. With these expressions, we can thoroughly analyze the effect of the parameter choice on the in-band and out-of-band radiation, but they are also useful for other signal processing tasks such as those mentioned for CP-OFDM. Our simulation results show that the proposed expressions are accurate. With the 
obtained expressions, we investigate the in-band and out-ofband radiation of UW-OFDM when using two well-known redundant carrier placement algorithms.

\section{SySTEMATIC UW-OFDM MODEL}

For generating the data sequence of the systematic coded UW-OFDM, the two-step approach is used [3]. In a block of $N$ time-domain samples characterized by $\mathbf{x}=\left[\mathbf{x}_{d}^{T} \mathbf{x}_{u}^{T}\right]^{T}$, the first $N-N_{u}$ samples, $\mathbf{x}_{d} \in \mathbb{C}^{N_{d} \times 1}$, are random and depend on the data and the last $N_{u}$ samples, $\mathbf{x}_{u} \in \mathbb{C}^{N_{u} \times 1}$, contain the known sequence which is called the unique word. To be able to add the unique word, in the first step, a block of $N_{u}$ zeroes in the time domain is generated by replacing $N_{r}$ data carriers by redundant carriers, where $N_{r} \geq N_{u}$. In the frequency domain, consider $\tilde{\mathbf{d}} \in \mathbb{C}^{N_{d} \times 1}$ as the vector of $N_{d}$ modulated data symbols that are independent and identically distributed $\left(E\left\{\tilde{\mathbf{d}} \tilde{\mathbf{d}}^{H}\right\}=\mathbf{I}_{N_{d}}\right)$ with zero mean $\left(E\{\tilde{\mathbf{d}}\}=\mathbf{0}_{N_{d} \times 1}\right)$. The vector of redundant data symbols is $\tilde{\mathbf{r}} \in \mathbb{C}^{N_{r} \times 1}$. As in conventional OFDM systems, $\left(N-N_{d}-N_{r}\right)$ zero subcarriers are inserted at DC and band edges. So, the frequency domain data vector is given by

$$
\tilde{\mathbf{x}}=\mathbf{B P}\left[\begin{array}{l}
\tilde{\mathbf{d}} \\
\tilde{\mathbf{r}}
\end{array}\right]
$$

where $\mathbf{P} \in \mathbb{R}^{\left(N_{d}+N_{r}\right) \times\left(N_{d}+N_{r}\right)}$ is the permutation matrix, which determines the positions of the data and redundant carriers, and $\mathbf{B} \in \mathbb{R}^{N \times\left(N_{d}+N_{r}\right)}$ inserts the zero subcarriers. In the systematic UW-OFDM system, the permutation matrix is calculated such that the energy allocated to redundant carriers is minimized. After IDFT and before insertion of the unique word, the time-domain data vector, $\mathbf{x}^{\prime}$, can be written as

$$
\mathbf{x}^{\prime}=\mathbf{F}_{N}^{-1} \mathbf{B P}\left[\begin{array}{l}
\tilde{\mathbf{d}} \\
\tilde{\mathbf{r}}
\end{array}\right]=\left[\begin{array}{c}
\mathbf{x}_{d} \\
\mathbf{0}
\end{array}\right] .
$$

where $\mathbf{F}_{N}^{-1}$ is the $N \times N$ IDFT matrix. The redundant carriers should be calculated so as to have $N_{u}$ zeros at the tail of the time-domain data vector, $\mathbf{x}^{\prime}$ (note that $\mathbf{x}=\mathbf{x}^{\prime}+\left[\mathbf{0}^{T} \mathbf{x}_{u}^{T}\right]^{T}$ ). To do so, a transform matrix is defined as $\mathbf{M}=\mathbf{F}_{N}^{-1} \mathbf{B P}$, which can be decomposed as follows

$$
\mathbf{M}=\left[\begin{array}{ll}
\mathbf{M}_{11} & \mathbf{M}_{12} \\
\mathbf{M}_{21} & \mathbf{M}_{22}
\end{array}\right]
$$

where $\mathbf{M}_{11}$ is $\left(N-N_{u}\right) \times N_{d}, \mathbf{M}_{12}$ is $\left(N-N_{u}\right) \times N_{r}$, $\mathbf{M}_{21}$ is $N_{u} \times N_{d}$ and $\mathbf{M}_{22}$ is $N_{u} \times N_{r}$. We should have $\mathbf{M}_{21} \tilde{\mathbf{d}}+\mathbf{M}_{22} \tilde{\mathbf{r}}=\mathbf{0}$. By solving this set of linear equations, the redundant carrier symbols can be determined by the linear mapping

$$
\tilde{\mathbf{r}}=-\mathbf{M}_{22}^{\dagger} \mathbf{M}_{21} \tilde{\mathbf{d}}=\mathbf{T} \tilde{\mathbf{d}}
$$

where $\mathbf{M}_{22}^{\dagger}=\mathbf{M}_{22}^{H}\left(\mathbf{M}_{22} \mathbf{M}_{22}^{H}\right)^{-1}$ is the Penrose-Moore pseudo-inverse. The redundant carrier symbols and the energy assigned to them depend on the choice of $\mathbf{P}$ through $\mathbf{M}$. By using (4) in (1), it can be seen that the redundant carriers introduce correlations in the frequency domain data vector $\tilde{\mathbf{x}}$. Different redundant carrier placement approaches are introduced such that the energy spent on redundant carriers is minimized [15], [16].

\section{PSD OF THE DFT BASED IMPLEMENTATION OF UW-OFDM SYSTEMS}

In this section, we propose an analytical expression for the power spectral density of the DFT-based implementation of the systematic coded UW-OFDM system. Assume that the $l$ th time-domain signal vector of the UW-OFDM system after insertion of the unique word be $\mathbf{x}^{(l)}=\left[x_{0}^{(l)}, x_{1}^{(l)}, \ldots, x_{N-1}^{(l)}\right]^{T}$. The consecutive time-domain signal vectors are converted to a discrete-time data sequence, $a[i]=x_{n}^{(l)}$, where $i=l N+n$, and $x_{n}^{(l)}$ is the $n$th element of $\mathbf{x}^{(l)}$. Before upconversion, this baseband sequence is passed through a D/A converter with an interpolation filter $g_{I}(t)$. The resulting analog signal is given by

$$
x(t)=\sum_{i=-\infty}^{+\infty} a[i] g_{I}\left(t-i T_{s}\right) .
$$

It has been discussed in [1] that the PSD of a D/A converted signal of the above form equals

$$
P(f)=\frac{\left|G_{I}(f)\right|^{2}}{T_{s}}\left[R(0)+2 \sum_{m=1}^{\infty} R(m) \cos \left(2 \pi f m T_{s}\right)\right],
$$

where $G_{I}(f)$ is the Fourier-transform of $g_{I}(t)$, and $T_{s}$ is the sampling time. This representation is based on the assumption that the discrete time data sequence $\{a[i]\}_{i=-\infty}^{+\infty}$ is stationary and has an autocorrelation function $R(i, m)=R(m) \triangleq$ $E\left\{a^{*}[i] a[i+m]\right\}$. In multicarrier transmission schemes, the discrete-time data sequence, $a[i]$, is a cyclostationary process. For example, in the systematic UW-OFDM system, the i.i.d. modulated data symbols are transmitted in consecutive frames at which a similar mapping is performed, e.g. as in (2). So, after D/A conversion, the correlation pattern imposed by this linear mapping is cyclically repeated. In [1], [13], it is proposed to time-average the autocorrelation function over one cyclic period to obtain the average PSD. By assuming that the frequency-domain data samples on all subcarriers are i.i.d., an approximate closed form expression for the PSD of CPOFDM and ZP-OFDM is determined in [1]. However, in the UW-OFDM system, the frequency-domain data samples on the subcarriers are correlated, such that a closed form solution is hard to obtain. Therefore, we describe the PSD of UW-OFDM in another way to tackle the inherent data correlation in UWOFDM frequency domain data vectors. The analog signal in (5) can be rewritten in a frame-based representation as follows

$$
x(t)=\sum_{l=-\infty}^{+\infty} \sum_{n=0}^{N-1} x_{n}^{(l)} g_{I}\left(t-(l N+n) T_{s}\right) .
$$

Consider the $l$ th data frame at the output of the IDFT module. Assume that the samples of this frame of data are known and repeated in all the frames. In other words, the signal in (7) is assumed to be periodic. The discrete-time Fourier transform (DTFT) of this frame is given by

$$
X^{(l)}(f)=\sum_{n=0}^{N-1} x_{n}^{(l)} e^{-j n 2 \pi f T_{s}} .
$$


After passing through the D/A interpolation filter, the power spectral density can be written as

$$
P(f)=\frac{\left|G_{I}(f)\right|^{2}}{T_{s}}\left|X^{(l)}(f)\right|^{2} .
$$

In reality, the modulated data symbols sent on different UWOFDM frames are different but they can be assumed to be independent. The correlation pattern of the data symbols in the frame is the same for all the frames. Therefore, although the sequence $x(t)$ in (7) is not periodic, it is statistically periodic, i.e., it is cyclostationary. Consequently, we will replace $\left|X^{(l)}(f)\right|^{2}$ by its statistical average over all possible realizations. In this way, the PSD of $x(t)$ is given as

$$
P(f)=\frac{\left|G_{I}(f)\right|^{2}}{T_{s}} E\left[\left|X^{(l)}(f)\right|^{2}\right],
$$

For obtaining a closed-form expression, we use the vectorform representation of (10). The DTFT values in (8) can be written in the vector form as follows

$$
X^{(l)}(f)=\mathbf{e}(f) \mathbf{x}^{(l)}=\mathbf{e}(f) \mathbf{F}^{H} \mathbf{P}\left[\begin{array}{l}
\mathbf{I} \\
\mathbf{T}
\end{array}\right] \tilde{\mathbf{d}},
$$

where $\mathbf{e}(f)$ is a $1 \times N$ row vector with the $n$th entry $e^{-j n 2 \pi f T}$. So, the PSD in (10) can be rewritten as

$$
P(f)=\frac{\left|G_{I}(f)\right|^{2}}{T_{s}} P_{1}(f),
$$

where

$$
\begin{aligned}
P_{1}(f) & =E\left[\left|X^{(l)}(f)\right|^{2}\right] \\
& =E\left\{\mathbf{e}(f) \mathbf{x}^{(l)} \mathbf{x}^{(l)^{H}} \mathbf{e}^{H}(f)\right\},
\end{aligned}
$$

which can be calculated as follows

$$
\begin{aligned}
P_{1}(f) & =E\left\{\mathbf{e}(f) \mathbf{F}^{H} \mathbf{P}\left[\begin{array}{c}
\mathbf{I} \\
\mathbf{T}
\end{array}\right] \tilde{\mathbf{d}} \tilde{\mathbf{d}}^{H}\left[\begin{array}{c}
\mathbf{I} \\
\mathbf{T}
\end{array}\right]^{H} \mathbf{P}^{H} \mathbf{F} \mathbf{e}^{H}(f)\right\} \\
& =\mathbf{e}(f) \mathbf{F}^{H} \mathbf{P}\left[\begin{array}{cc}
\mathbf{I} & \mathbf{T}^{H} \\
\mathbf{T} & \mathbf{T} \mathbf{T}^{H}
\end{array}\right] \mathbf{P}^{H} \mathbf{F} \mathbf{e}^{H}(f) .
\end{aligned}
$$

Equation (12) consists of two components. The first component is $\left|G_{I}(f)\right|^{2}$ which is the frequency response of the interpolation filter with cut-off frequency $1 / 2 T_{s}$; this is desired to be as flat as possible in the in-band part of the spectrum, and to have a sharp spectral roll-off so as to suppress the out-of-band radiation of the system very sharply. The second component, $P_{1}(f)$, depends completely on the characteristics of the data to be transmitted. As it can be seen from (14), $P_{1}(f)$ is periodic with a period $1 / T_{s}$. Therefore, the interpolation filter needs to reject the unnecessary OOB parts of the UW-OFDM spectrum caused by the periodicity of the signal.

\section{Simulation Results}

In this section, the validity of the proposed PSD expression is investigated by comparing with the estimated PSD attained by simulations. Also, the spectral characteristics of UW-OFDM system are compared with those of CP-OFDM.
TABLE I

MAIN PHY PARAMETERS OF THE INVESTIGATED SYSTEMS

\begin{tabular}{|l|c|c|}
\hline & CP-OFDM & UW-OFDM \\
\hline \hline Occupied subcarriers & 52 & 52 \\
\hline Data subcarriers & 48 & 36 \\
\hline Additional subcarriers & 4 (pilots) & 16 (redundant) \\
\hline DFT period & $3.2 \mu \mathrm{s}$ & $3.2 \mu \mathrm{s}$ \\
\hline Guard duration & $800 \mathrm{~ns}$ & $800 \mathrm{~ns}$ \\
\hline Total OFDM symbol duration & $4 \mu \mathrm{s}$ & $3.2 \mu \mathrm{s}$ \\
\hline Bandwidth & $20 \mathrm{MHz}$ & $20 \mathrm{MHz}$ \\
\hline Modulation scheme & $16 \mathrm{QAM}$ & $16 \mathrm{QAM}$ \\
\hline
\end{tabular}

For the simulations, a sequence of uncoded independent and identically distributed data bits are modulated with 16QAM modulation. Then, they are passed to a UW-OFDM transmitter with $N=64$ subcarriers. Similar to [4], the UWOFDM specifications are taken from the IEEE 802.11a WLAN standard [17], and are given in Table I. The indices of the zero subcarriers in the frequency domain for both systems are $\{0,27,28, \ldots, 37\}$. The indices of the redundant carriers are, unless otherwise stated, obtained by maximum distance algorithm [18]. The unique word is considered to be zero. The simulated PSDs are obtained by using Welch's periodogram algorithm [19]. In Figure 1, the proposed analytical expression for the PSD of the UW-OFDM before the interpolation filter (14) is shown by the dotted curve. The estimated PSDs of the simulated UW-OFDM and CP-OFDM and the analytical expression developed for CP-OFDM without zero subcarriers (w/o ZS) [1] are included for comparison. The PSD expression in [1] is developed on the basis of the assumption that the data on all subcarriers are independent, and no zero subcarriers are inserted in the frequency domain. All the curves are normalized by the peak values. It can be seen from the zoomed view that the UW-OFDM PSD curve corresponding to the analytical expression is almost the same as the estimated PSD curve.

One of the shortcomings of the CP-OFDM transmission scheme is that the power of sidelobes decay slowly in the out-of-band. This can cause spectral interference to systems operating in the adjacent bands. However, the analytical and estimated PSD of UW-OFDM show that the OOB radiation of UW-OFDM is much lower than the OOB radiation of CPOFDM. This is one of the advantages of UW-OFDM over CP-OFDM.

The other parameter affecting the OOB radiation of multicarrier systems is the interpolation low pass filter. This effect is investigated in Figure 2 by showing the normalized PSD after interpolation lowpass filter. Note that the PSD before the interpolation filter is periodic and repeated with period $1 / T_{s}=20 \mathrm{MHz}$. After the interpolation filter, the spectral leakage of both the systems is directly controlled by the spectral leakage of the interpolation filter, $G_{I}(f)$. In this figure, the data sequences of both systems are upsampled with a factor of 2. The interpolation filter is a 22nd-order equiripple finite impulse response filter with $1 \mathrm{~dB}$ passband ripple and a stopband attenuation of $60 \mathrm{~dB}$. The analytical 


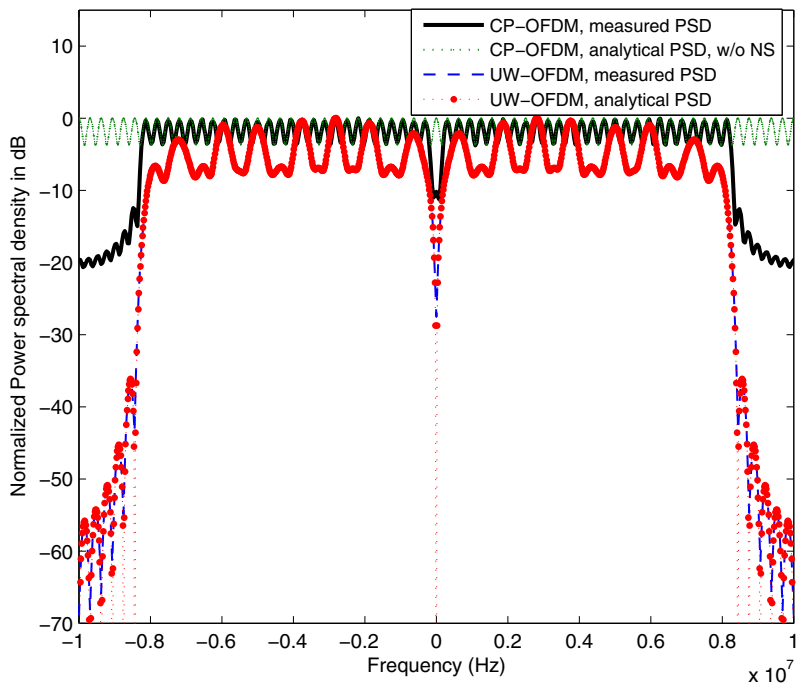

(a)

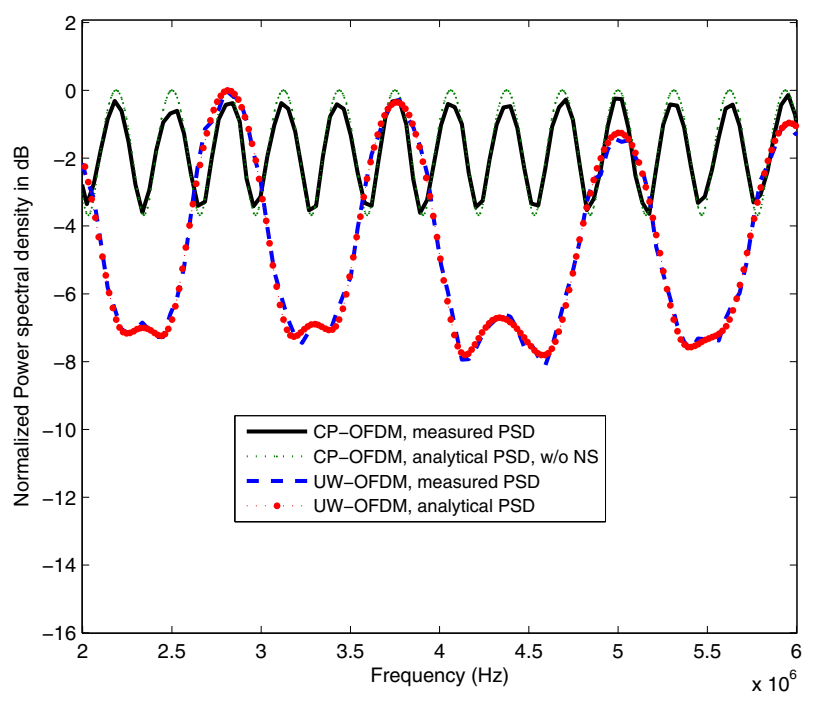

(b)

Fig. 1. Normalized power spectral density comparison before the low-pass interpolation filter: (a) full view and (b) zoom on part of in-band spectrum.

PSD for UW-OFDM is obtained from (12) by multiplying the frequency response of the interpolation filter with $P_{1}(f)$, and it is almost the same as the estimated PSD. This shows the accuracy of the proposed PSD relations for modeling the spectral behavior of the UW-OFDM after LPF. The high rolloff factor of the unique word OFDM combined with the OOB reduction capability of the LPF results in a very sharp response in the OOB compared to the slow decaying of the CP-OFDM in the OOB.

The total performance of the systematic UW-OFDM completely depends on the selection of the position of the redundant carriers. In Figure 3, the PSD of the UW-OFDM data sequence before upsampling and interpolation filtering

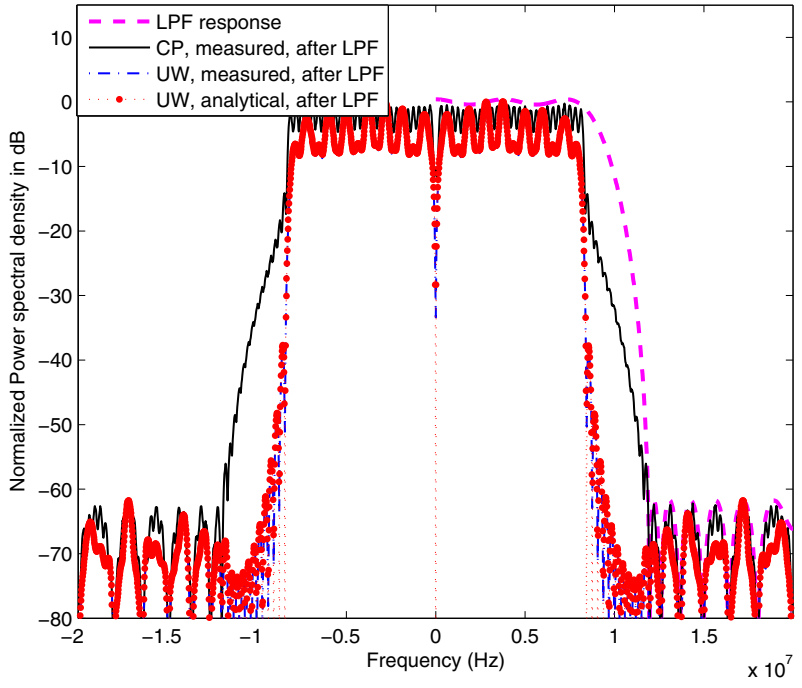

(a)

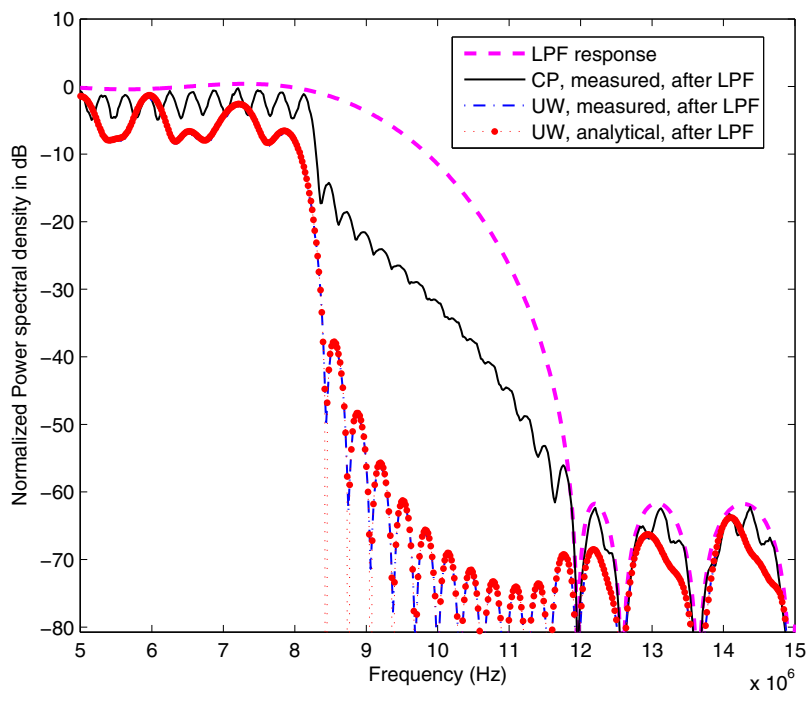

(b)

Fig. 2. The effect of interpolation LPF on the analytical and measured PSD curves: (a) full view and (b) zoom on part of in-band and OOB spectrum.

is depicted based on (14) for two redundant carrier placement approaches, i.e. maximum distance (MD) placement and heuristic algorithm (HE) [18]. Different redundant carrier placement approaches need different powers to be allocated to the redundant carriers. We have assumed that the power of i.i.d. modulated data symbols is $E_{s}=1$ for UW-OFDM system, i.e., we have $E\left\{\tilde{\mathbf{d}} \tilde{\mathbf{d}}^{H}\right\}=\mathbf{I}_{N_{d}}$. So, for the UW-OFDM, the power allocated to $N_{d}$ data symbols is $P_{d} / E_{s}=N_{d}=36$. In [15], it is shown that the minimum power required to be allocated to the redundant carriers is $\min \left\{P_{r} / E_{s}\right\}=$ $N_{d}=36$. When the redundant carriers are placed by the near-optimal heuristic algorithm, the required power for the $\mathrm{RCs}$ is $P_{r, H E} / E_{s} \approx 36.2$ which is quite near the optimal 


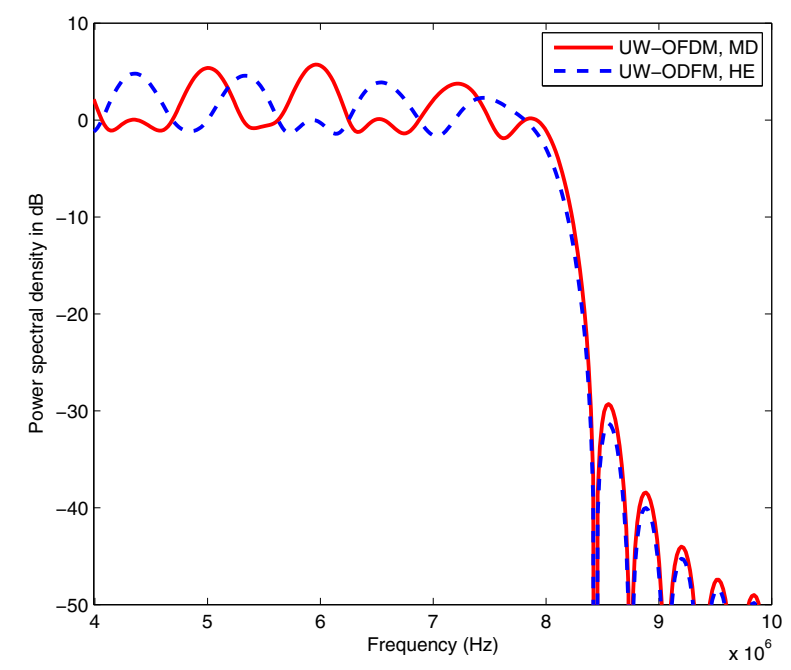

Fig. 3. Power spectral density comparison for different redundant carrier placement approaches.

value. In contrast, the power needed for MD algorithm is $P_{r, M D} / E_{s} \approx 52.3$. In Figure 3, the zero crossing points in the PSD of the UW-OFDM system are the data subcarriers with unit norm and the in-band peaks represent the redundant carriers. It is seen that the in-band peak values for UWOFDM with the MD placement approach are as expected higher compared to that of heuristic algorithm, i.e. higher redundant energy is needed. Further, it can be seen in the figure that the heuristic approach not only outperforms the MD algorithm regarding the lower power needed for the placement of redundant carriers, but it also has $2 \mathrm{~dB}$ lower OOB radiation.

\section{CONClusion}

One of the main performance characteristics of communication transmission schemes is the transmitted power spectral density. In this paper, the characterization of the PSD of the systematic coded UW-OFDM is addressed. In this system, the insertion of redundancy in the frequency domain creates correlation in the UW-OFDM frame. So, the conventional spectral modeling approaches that assume the independency of the frequency domain data vectors are not applicable for UWOFDM systems. However, the correlation pattern is repeated at each UW-OFDM frame making the time domain data sequence be cyclostationary. The power spectral density is derived by calculating the Fourier transform of one of the frame samples and averaging its squared magnitude over all UW-OFDM frames. The simulation results have shown the accuracy of the derived expression. It is shown in this paper that one of the important characteristics of UW-OFDM is that the OOB radiation is much lower than the CP-OFDM system. So, it is a good candidate for situations that need low out-of-band radiation such as dynamic spectrum access in cognitive radio networks. Finally, it is shown that redundant carrier placement approaches that require lower redundant energy, also have slightly lower OOB radiation.

\section{ACKNOWLEDGMENT}

This research has been funded by the Interuniversity Attraction Poles Programme initiated by the Belgian Science Policy Office. The authors want to thank Prof. Mario Huemer and Mr. Christian Hofbauer for the help on the simulations and Prof. Marc Moonen and Dr. Toon van Waterschoot for the helpful discussions.

\section{REFERENCES}

[1] T. van Waterschoot, V. Le Nir, J. Duplicy, and M. Moonen, "Analytical expressions for the power spectral density of CP-OFDM and ZP-OFDM signals," IEEE Signal Processing Letters, vol. 17, no. 4, pp. 371-374, 2010.

[2] H. Steendam and M. Moeneclaey, "Different guard interval techniques for OFDM: performance comparison," Multi-Carrier Spread Spectrum 2007, pp. 11-24, 2007.

[3] A. Onic and M. Huemer, "Direct vs. two-step approach for unique word generation in UW-OFDM," in International OFDM Workshop, 2010, pp. $285-299$.

[4] M. Huemer, C. Hofbauer, and J. Huber, "Non-systematic complex number RS coded OFDM by unique word prefix," Signal Processing, IEEE Transactions on, vol. 60, no. 1, pp. 285 -299, Jan. 2012.

[5] C. Liu and F. Li, "Spectrum modelling of OFDM signals for WLAN," IEEE Electronics Letters, vol. 40, no. 22, pp. 1431-1432, 2004.

[6] S. Talbot and B. Farhang-Boroujeny, "Spectral method of blind carrier tracking for OFDM," Signal Processing, IEEE Transactions on, vol. 56, no. 7, pp. 2706-2717, 2008.

[7] M. Pauli and P. Kuchenbecker, "On the reduction of the out-of-band radiation of OFDM signals," in IEEE International Conference on Communications, vol. 3, 1998, pp. 1304-1308.

[8] I. Cosovic, S. Brandes, and M. Schnell, "Subcarrier weighting: a method for sidelobe suppression in OFDM systems," IEEE Communications Letters, vol. 10, no. 6, pp. 444-446, 2006.

[9] H. Mahmoud and H. Arslan, "Sidelobe suppression in OFDM-based spectrum sharing systems using adaptive symbol transition," IEEE Communications Letters, vol. 12, no. 2, pp. 133-135, 2008.

[10] M. Ma, X. Huang, B. Jiao, and Y. Guo, "Optimal orthogonal precoding for power leakage suppression in DFT-based systems," Communications, IEEE Transactions on, vol. 59, no. 3, pp. 844-853, 2011.

[11] J. Zhang, X. Huang, A. Cantoni, and Y. Guo, "Sidelobe suppression with orthogonal projection for multicarrier systems," Communications, IEEE Transactions on, vol. 60, no. 2, pp. 589-599, 2012.

[12] Y. Lin and S. Phoong, "OFDM transmitters: analog representation and DFT-based implementation," Signal Processing, IEEE Transactions on, vol. 51, no. 9, pp. 2450-2453, 2003.

[13] M. Ivrlač and J. Nossek, "Influence of a cyclic prefix on the spectral power density of cyclo-stationary random sequences," Multi-Carrier Spread Spectrum 2007, pp. 37-46, 2007.

[14] S.-G. Huang and C.-H. Hwang, "Improvement of active interference cancellation: avoidance technique for OFDM cognitive radio," Wireless Communications, IEEE Transactions on, vol. 8, no. 12, pp. 5928-5937, 2009.

[15] H. Steendam, "Analysis of the redundant energy in UW-OFDM," Communications, IEEE Transactions on, vol. 60, no. 6, pp. 1692 -1701, June 2012.

[16] $\longrightarrow$, "On the redundant carrier distribution for UW-OFDM," in IEEE Wireless Communications and Networking Conference (WCNC), April 2012, pp. $32-36$.

[17] Wireless LAN Medium Access Control (MAC) and Physical Layer (PHY) Specifications: High-Speed Physical Layer in the $5 \mathrm{GHz}$ Band, IEEE Std. 802.11a-1999(R2003), Sep. 1999. Std.

[18] H. Steendam, "On the selection of the redundant carrier positions in UW-OFDM," Signal Processing, IEEE Transactions on, vol. 61, no. 5, pp. 1112-1120, March 2013.

[19] P. Welch, "The use of fast Fourier transform for the estimation of power spectra: a method based on time averaging over short, modified periodograms," Audio and Electroacoustics, IEEE Transactions on, vol. 15 , no. 2, pp. 70-73, 1967. 